nephron

Practice
Nephron 2019;143:43-53

DOI: $10.1159 / 000500485$
Received: October 17, 2018

Accepted after revision: April 16, 2019

Published online: June 11, 2019

\title{
Relationship between History of Ischemic Stroke and All-Cause Mortality in Incident Dialysis Patients
}

\author{
Masayasu Kojima Daijo Inaguma Shigehisa Koide Eri Koshi-Ito \\ Kazuo Takahashi Hiroki Hayashi Naotake Tsuboi Midori Hasegawa \\ Yukio Yuzawa \\ Department of Nephrology, Fujita Health University School of Medicine, Toyoake, Japan
}

\section{Keywords \\ Stroke $\cdot$ Chronic kidney disease - Dialysis initiation · \\ Mortality · Propensity score}

\begin{abstract}
Background: Few studies have focused on the association between history of ischemic stroke at predialysis stage and mortality after dialysis initiation. Objective: To examine whether history of stroke in incident dialysis patients is associated with mortality, including all-cause and cardiovascu$\operatorname{lar}(\mathrm{CV})$-related mortality. Methods: The study database was derived from the Aichi Cohort Study of Prognosis in Patients Newly Initiated into Dialysis, a multicenter, prospective, cohort analysis. We classified patients into 2 groups according to their history of ischemic stroke and compared their outcomes. Propensity scores (PSs) represented the probability of being assigned to a group with or without a history of ischemic stroke. We defined the following outcomes: allcause mortality; CV-related mortality; non-CV-related mortality; infection-related mortality; and stroke event after dialysis initiation. Factors contributing to the outcomes were
\end{abstract}

\begin{tabular}{ll}
\hline KARGER & $\begin{array}{l}\text { (c) } 2019 \text { The Author(s) } \\
\text { Published by S. Karger AG, Basel }\end{array}$ \\
E-Mail karger@karger.com & This article is licensed under the Creative Commons Attribution- \\
www.karger.com/nef & $\begin{array}{l}\text { NonCommercial-NoDerivatives 4.0 International License (CC BY- } \\
\text { NC-ND) (http://www.karger.com/Services/OpenAccessLicense). } \\
\text { Usage and distribution for commercial purposes as well as any dis- } \\
\text { tribution of modified material requires written permission. }\end{array}$
\end{tabular}

examined using stepwise multivariate Cox proportional hazards analysis. Results: All-cause mortality was significantly higher in the ischemic stroke group (log-rank test $p<0.001$ ). All-cause, non-CV-related, and infection-related mortality and stroke event after dialysis initiation were significantly higher in the ischemic stroke group after PS matching (logrank test: $p<0.001,<0.001,0.002$, and 0.002, respectively). History of ischemic stroke was associated with all-cause mortality in univariate analysis (hazard ratio [HR] $1.85,95 \% \mathrm{Cl}$ 1.44-2.37). History of ischemic stroke before dialysis initiation was associated with all-cause mortality in multivariate analysis (HR 1.39, 95\% Cl 1.05-1.85). Conclusion: The present study revealed that history of ischemic stroke before dialysis initiation was associated with all-cause, non-CV-related, and infection-related mortality and stroke event after dialysis initiation during maintenance dialysis.

(C) 2019 The Author(s)

Published by S. Karger AG, Basel

D.I. and S.K.: The Aichi Cohort Study of Prognosis in Patients Newly Initiated into Dialysis (AICOPP) Group.
Daijo Inaguma, MD

Department of Nephrology, Fujita Health University

Dengakugakubo 1-98, Kutsukake

Aichi, Toyoake 470-1192 (Japan)

E-Mail address daijo@ fujita-hu.ac.jp 


\section{Introduction}

Chronic kidney disease (CKD) is considered one of the most serious medical issues because it not only progresses to end-stage kidney disease but also leads to cardiovascular (CV) events and death [1-5]. Generally, the risk factors for stroke onset were as follows: aging, hypertension, lipid profile disorder, and CKD incidence and progression. In particular, both stroke and CKD patients have an equal risk of death [6]. Ovbiagele et al. [7] reported that patients with CKD accounted for 35\% of patients with ischemic stroke in a large-scale cohort. Furthermore, Del Fabbro et al. [8] reported that out of 890 patients, 82 patients died 1 year after hospital discharge, $61 \%$ of whom had CKD. The onset of stroke in patients with predialysis CKD is related to the decline in kidney function and the survival rate [9]. Similarly, the onset of stroke in dialysis patients leads to a reduction in activities of daily living (ADL) and an increase in the mortality rate [10].

CKD sequentially progresses from the predialysis stage via dialysis initiation to maintenance dialysis. From this perspective, we presumed that certain conditions and methods of disease management during the predialysis stage possibly influenced the incidence of CV events and mortality [11]. However, few studies have focused on the association between history of ischemic stroke at the predialysis stage and mortality after dialysis initiation.

Therefore, we examined the association between history of stroke in incident dialysis patients and mortality, including all-cause and CV-related mortality, using the database of incident dialysis patients.

\section{Materials and Methods}

\section{Subjects}

The study database was derived from the Aichi Cohort Study of Prognosis in Patients Newly Initiated into Dialysis (AICOPP), which was a multicenter, prospective cohort at the 17 AICOPP group centers between October 2011 and September 2016 [12]. Dialysis was initiated in 1,889 consecutive patients. We excluded 369 patients who died during hospitalization for dialysis initiation, withdrew from maintenance dialysis, or refused registration. The patients enrolled were at least 20 years old, had end-stage kidney disease, and gave written informed consent. In total, 1,520 patients were enrolled.

Baseline Patient Characteristics and Laboratory Data

Baseline was defined as the time at which dialysis was initiated. The blood pressure and body mass index (BMI) were measured prior to the first dialysis session. Coronary artery disease (CAD) was defined as follows: (1) history of percutaneous coro- nary artery intervention or coronary artery bypass graft, (2) episode of ischemic change on electrocardiogram with symptoms, and (3) positive findings during diagnostic imaging such as coronary artery computed tomography and myocardial scintigraphy. Symptoms of heart failure at dialysis initiation were defined as follows: (1) dyspnea accompanied with hypoxemia, (2) pulmonary congestion and pleural effusion on chest radiography, and (3) physical examination findings indicating fluid retention (edema and weight gain and jugular vein distention). Diabetes mellitus (DM) was defined as follows: (1) fasting blood glucose level $\geq 126 \mathrm{mg} / \mathrm{dL}$, (2) random blood glucose level $\geq 200 \mathrm{mg} / \mathrm{dL}$, (3) hemoglobin A1c (National Glycohemoglobin Standardization Program) level $\geq 6.5 \%$, and (4) the use of insulin or oral hypoglycemic agents. The Barthel index (BI) is one of the most widely employed measurement scales for the evaluation of functionality [13]. The evaluation has a total score of 100 , based on 10 items with a possible 10 points each. Thus, the highest score is 100 and the lowest is 0 . A score of 100 points indicates an independent daily life. Since all the patients were started on dialysis during hospitalization for a few weeks, the BI was assessed just before hospital discharge or transfer to another facility, because physical function at dialysis initiation did not reflect normal status due to uremia.

\section{Definition of Stroke}

History of stroke, including both ischemic and hemorrhagic types, was defined as hospitalization for stroke treatment or obvious positive findings on diagnostic imaging, including computed tomography and magnetic resonance imaging. The patients were classified into 2 groups: those with and without a history of ischemic stroke, that is, the ischemic stroke and non-stroke groups. Patients with a history of hemorrhagic stroke were excluded from both groups.

\section{Outcomes}

We categorized the outcomes into 5 types: (1) all-cause mortality, (2) CV-related mortality, (3) non-CV-related mortality, (4) infection-related mortality, and (5) stroke event after dialysis initiation. We compared the outcomes between the ischemic stroke and non-stroke groups. The following were defined as CV disease: (1) heart failure requiring hospitalization, (2) acute coronary syndrome, (3) stroke, and (4) peripheral artery disease requiring hospitalization. Heart failure was diagnosed on the basis of hypoxemia with pulmonary congestion, pulmonary edema, or pleural effusion on chest radiography. Acute coronary syndrome was diagnosed on the basis of stenosis or occlusion on coronary angiography, percutaneous coronary artery intervention, or coronary artery bypass graft or electrocardiographic findings consistent with acute coronary syndrome. Stroke was diagnosed on the basis of neurological symptoms along with brain computed tomography or magnetic resonance imaging findings indicative of hemorrhage or infarction. We defined CV-related mortality as death due to the aforementioned CV diseases, and infection-related mortality as death due to complications such as sepsis, pneumonia, and urinary tract infection.

\section{Stratified Analysis}

Neuromuscular symptoms associated with stroke, such as hemiparesis, affect physical function. We surmised that impaired physical function might lead to low patient survival. According- 
Table 1. Comparison of baseline characteristics and laboratory data between the ischemic stroke group and the non-stroke group

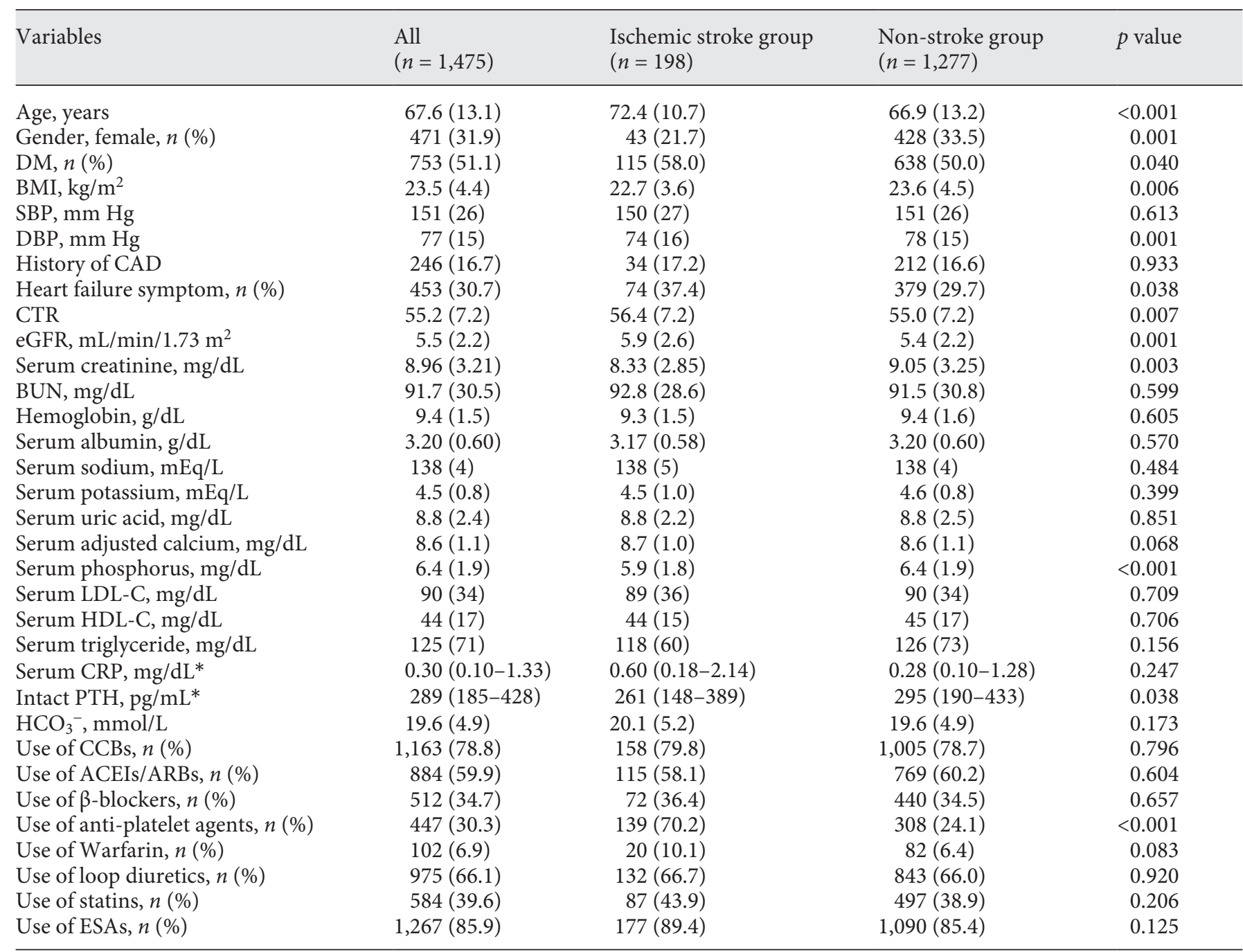

Values are expressed as mean (SD).

* Median (interquartile range).

BMI, body mass index; SBP, systolic blood pressure; DBP, diastolic blood pressure; CAD, coronary artery disease; CTR, cardiothoracic ratio; eGFR, estimated glomerular filtration rate; $\mathrm{BUN}$, blood urea nitrogen; LDL-C, low-density lipoprotein cholesterol; HDL-C, high-density lipoprotein cholesterol; CRP, C-reactive protein; PTH, parathyroid hormone; CCB, calcium channel blocker; ACEI, angiotensin converting enzyme inhibitor; $\mathrm{ARB}$, angiotensin receptor blocker; ESA, erythropoiesis stimulating agent; DM, diabetes mellitus.

ly, we conducted stratified analysis. We classified the patients into 2 groups, those with a BI score of 100 and those with a score $<100$, and compared the outcomes between the 2 groups.

\section{Prognosis Surveillance}

We surveyed the outcomes at 3 time points: April 30, 2014; April 30, 2015; and September 30, 2016. Outcome data including all-cause mortality were obtained from the medical records of the AICOPP group institutions or responses from letters sent to facilities where the patients had been transferred for maintenance dialysis.

Stroke and Mortality in Incident Dialysis

Patients

\section{Statistical Analysis}

SPSS Statistics version 25 and the Easy R program were used for statistical analysis [14]. Baseline characteristics and laboratory data at the first dialysis session were compared between the ischemic stroke and non-stroke groups using unpaired $t$ test and Fisher's exact test for nominal variables. All-cause mortality in the entire cohort was compared between the ischemic stroke and non-stroke groups by using the log-rank test for Kaplan-Meier curves. Factors related to all-cause mortality were selected using a univariate Cox proportional hazards regression analysis for all cases. We employed the following models for multivariate anal- 
Fig. 1. Comparison of all-cause mortality between the 2 groups in the entire cohort. All-cause mortality was significantly higher in the ischemic stroke group (log-rank test $p<0.001$ ).

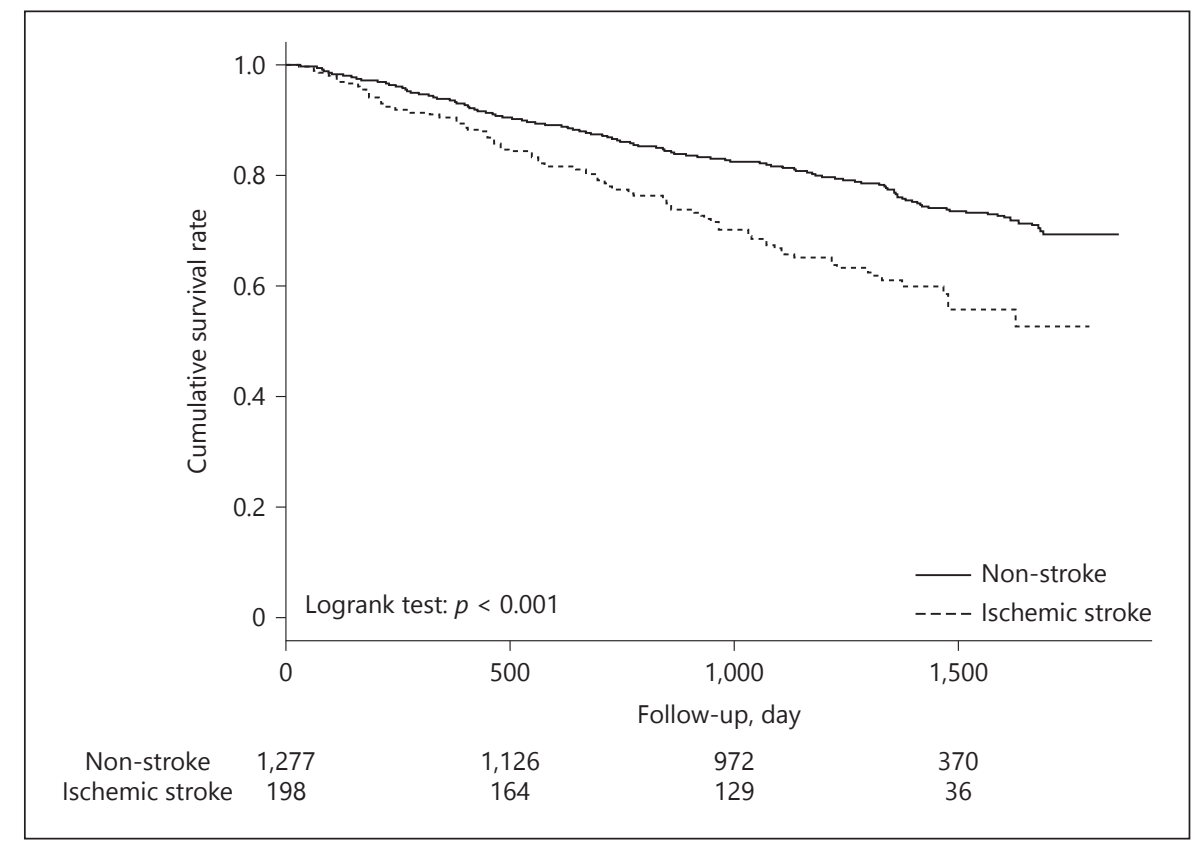

ysis: adjusted for age and sex (model 1); adjusted for model 1, with addition of DM, CAD, systolic blood pressure (SBP), and diastolic blood pressure (model 2); adjusted for model 2, with addition of hemoglobin, albumin, estimated glomerular filtration rate (eGFR), and low-density lipoprotein cholesterol (LDL- C; model 3); and adjusted for model 3, with addition of $\mathrm{BMI}$, cardiothoracic ratio (CTR), loop diuretics, calcium channel blockers (CCBs), statins, angiotensin receptor blockers (ARBs), and angiotensin-converting enzyme inhibitors (ACEIs; model 4). We also conducted a stepwise multivariate analysis.

The propensity score (PS) was calculated using logistic regression models by matching the background characteristics with age, sex, DM, and CAD. Then, analyses of all-cause mortality, CV-related mortality, non-CV-related mortality, infection-related mortality, and stroke event after dialysis initiation were conducted for the ischemic stroke and non-stroke groups after PS matching. Furthermore, we conducted subgroup analysis for the BI score after PS matching adjusted for age, sex, DM, and CAD and compared all-cause mortalities between the subgroup/group with a BI score of 100 and that with a BI score of $<100$. Statistical significance was defined as $p<0.05$.

\section{Results}

Comparison of Baseline Patient Characteristics and Laboratory Data between the Ischemic Stroke and Non-stroke Groups Comprising the

Entire Cohort

The incidence of ischemic stroke was greater than that of hemorrhage stroke (34 cases of hemorrhage stroke, 198 cases of ischemic stroke, and 11 cases of ischemic stroke with hemorrhage). Table 1 shows a comparison of the baseline patient characteristics and laboratory data between the ischemic stroke and non-stroke groups. Patients in the ischemic stroke group were significantly older and had a lower proportion of women, higher proportion of DM, lower BMI, lower DBP, higher proportion of heart failure symptoms, higher CTR, higher eGFR, lower serum creatinine level, lower serum phosphorus level, lower intact parathyroid hormone, and higher proportions of antiplatelet drug use and statin use than those in the non-stroke group. However, there were no significant differences in serum albumin and C-reactive protein between the ischemic stroke and non-stroke groups. The online supplementary table (see www.karger.com/ doi/10.1159/000500485) shows the comparison of baseline patient characteristics and laboratory data between the ischemic stroke and non-stroke groups comparing the entire cohort including patients with hemorrhagic stroke.

\section{Comparison of All-Cause Mortality between the Ischemic Stroke and Non-stroke Groups Comprising} the Entire Cohort

There were 381 cases $(25.8 \%)$ of all-cause mortality during the follow-up period (ischemic stroke group: 78, non-stroke group: 303). Figure 1 shows the comparison of all-cause mortality between the 2 groups. All-cause mortality was significantly higher in the ischemic stroke group (log-rank test $p<0.001$ ). 
Table 2. HR for all-cause mortality using a univariate Cox proportional analysis without hemorrhage

\begin{tabular}{lrrr}
\hline Variables & HR & $95 \%$ CI & $p$ value \\
\hline Ischemic stroke & 1.85 & $1.44-2.37$ & $<0.001$ \\
Age $(10$ years old) & 1.90 & $1.72-2.11$ & $<0.001$ \\
Gender, female & 0.65 & $0.51-0.82$ & $<0.001$ \\
DM & 1.02 & $0.83-1.24$ & 0.879 \\
BMI & 0.92 & $0.89-0.94$ & $<0.001$ \\
SBP $(10 \mathrm{~mm} \mathrm{Hg})$ & 0.94 & $0.91-0.97$ & $<0.001$ \\
DBP $(10 \mathrm{~mm} \mathrm{Hg)}$ & 0.85 & $0.81-0.89$ & $<0.001$ \\
History of CAD & 1.76 & $1.39-2.22$ & $<0.001$ \\
CTR & 1.03 & $1.02-1.04$ & $<0.001$ \\
eGFR & 1.11 & $1.08-1.15$ & $<0.001$ \\
Serum creatinine & 0.86 & $0.83-0.90$ & $<0.001$ \\
BUN (10 mg/dL) & 1.04 & $1.01-1.07$ & 0.018 \\
Hemoglobin & 0.93 & $0.87-0.99$ & 0.022 \\
Serum albumin & 0.66 & $0.56-0.77$ & $<0.001$ \\
Serum LDL-C $(10 \mathrm{mg} / \mathrm{dL})$ & 0.96 & $0.93-1.00$ & 0.041 \\
Serum HDL-C $(10 \mathrm{mg} / \mathrm{dL})$ & 0.95 & $0.88-1.02$ & 0.127 \\
Serum triglyceride $(10 \mathrm{mg} / \mathrm{dL})$ & 1.00 & $0.98-1.01$ & 0.782 \\
Serum CRP & 1.04 & $1.02-1.05$ & $<0.001$ \\
HCO ${ }^{-}$ & 1.02 & $1.00-1.05$ & 0.088 \\
Use of CCBs & 0.66 & $0.53-0.83$ & $<0.001$ \\
Use of ACEIs/ARBs & 0.71 & $0.58-0.86$ & $<0.001$ \\
Use of $\beta$-blockers & 1.13 & $0.92-1.40$ & 0.233 \\
Use of loop diuretics & 1.28 & $1.03-1.60$ & 0.027 \\
Use of statins & 0.81 & $0.65-0.99$ & 0.044 \\
Use of ESAs & 0.77 & $0.58-1.01$ & 0.059 \\
\hline
\end{tabular}

$\mathrm{HR}$, hazard ratio; DM, diabetes mellitus; $\mathrm{BMI}$, body mass index; SBP, systolic blood pressure; DBP, diastolic blood pressure; $\mathrm{CAD}$, coronary artery disease; CTR, cardiothoracic ratio; eGFR, estimated glomerular filtration rate; BUN, blood urea nitrogen; LDL-C, low-density lipoprotein cholesterol; HDL-C, high-density lipoprotein cholesterol; CRP, C-reactive protein; CCB, calcium channel blocker; ACEI, angiotensin converting enzyme inhibitor; $\mathrm{ARB}$, angiotensin receptor blocker; ESA, erythropoiesis stimulating agent.

\section{Univariate Analysis of Factors Affecting All-cause} Mortality in the Entire Cohort

We obtained factors affecting all-cause mortality by performing a univariate Cox proportional hazards regression analysis (Table 2). The history of ischemic stroke prior to dialysis initiation was associated with all-cause mortality (hazard ratio [HR] 1.85, 95\% CI 1.44- 2.37). Among other factors, age; sex; BMI; SBP; DBP; history of CAD; CTR; eGFR; serum creatinine level; blood urea nitrogen; hemoglobin level; serumalbumin; serum LDL-C; serum CRP; and use of CCBs, ACEIs/ ARBs, loop diuretics, and statins were also found to be associated with all-cause mortality.

Stroke and Mortality in Incident Dialysis Patients
Table 3. HR of ischemic stroke for all-cause mortality using a multivariate cox proportional analysis

\begin{tabular}{lllr}
\hline Variables & HR & $95 \%$ CI & $p$ value \\
\hline Unadjusted & 1.85 & $1.44-2.37$ & $<0.001$ \\
Model 1 & 1.42 & $1.10-1.82$ & 0.007 \\
Model 2 & 1.41 & $1.09-1.81$ & 0.008 \\
Model 3 & 1.48 & $1.12-1.96$ & 0.005 \\
Model 4 & 1.36 & $1.01-1.82$ & 0.042 \\
\hline
\end{tabular}

Model 1: Adjusted for age, and gender.

Model 2: Model 1 plus DM, CAD, SBP, and DBP.

Model 3: Model 2 plus Hb, Alb, eGFR and LDL-C.

Model 4: Model 3 plus BMI, CTR, Loop diuretics, CCB, Statin and ACEIARB.

$\mathrm{HR}$, hazard ratio; DM, diabetes mellitus; $\mathrm{CAD}$, coronary artery disease; SBP, systolic blood pressure, DBP, diastolic blood pressure; Hb, hemoglobin; Alb, albumin; eGFR, estimated glomerular filtration rate; LDL-C, low density lipoprotein cholesterol; BMI, body mass index; CTR, cardiothoracic ratio; CCB, calcium channel blocker; ACEI, angiotensin converting enzyme inhibitor; ARB, angiotensin receptor blocker.

Table 4. Model 4 HR for all-cause mortality by multivariate Cox proportional hazard analysis using the stepwise method

\begin{tabular}{lllr}
\hline Variables & HR & $95 \%$ CI & $p$ value \\
\hline Ischemic stroke & 1.39 & $1.05-1.85$ & 0.023 \\
Age $(10$ years old $)$ & 1.97 & $1.74-2.23$ & $<0.001$ \\
SBP $(10$ mm Hg) & 0.96 & $0.91-1.00$ & 0.040 \\
Serum albumin & 0.77 & $0.64-0.94$ & 0.010 \\
eGFR & 1.08 & $1.03-1.12$ & 0.001 \\
Use of CCBs & 0.64 & $0.49-0.85$ & 0.002 \\
\hline
\end{tabular}

$\mathrm{HR}$, hazard ratio; SBP, systolic blood pressure; eGFR, estimated glomerular filtration rate; CCB, calcium channel blocker.

\section{Multivariate Analysis of Factors Affecting All-cause Mortality in the Entire Cohort}

The history of ischemic stroke prior to dialysis initiation was associated with all-cause mortality adjusted for age, sex, DM, CAD, SBP, DBP, hemoglobin, albumin, eGFR, LDL-C, BMI, CTR, loop diuretics, CCBs, statin, and ACEIs/ARBs (HR 1.36, 95\% CI 1.01- 1.82; Table 3). Furthermore, stepwise multivariate analysis revealed a significant association between the history of ischemic stroke and all-cause mortality (HR 1.39, 95\% CI 1.051.85). In addition, age, SBP, serum albumin, eGFR, and use of CCBs were significantly associated with all-cause mortality (Table 4). 
Table 5. HR of ischemic stroke using a multivariate Cox proportional analysis

\begin{tabular}{lrrr}
\hline Variables & HR & $95 \%$ CI & $p$ value \\
\hline CV-related mortality & & & \\
$\quad$ Unadjusted & 1.71 & $1.14-2.58$ & 0.010 \\
$\quad$ Model 1 & 1.38 & $0.91-2.08$ & 0.130 \\
$\quad$ Model 2 & 1.33 & $0.88-2.02$ & 0.176 \\
$\quad$ Model 3 & 1.32 & $0.84-2.07$ & 0.230 \\
$\quad$ Model 4 & 1.21 & $0.76-1.94$ & 0.423 \\
Non-CV-related mortality & & & \\
$\quad$ Unadjusted & 1.86 & $1.34-2.59$ & $<0.001$ \\
$\quad$ Model 1 & 1.38 & $0.99-1.93$ & 0.056 \\
$\quad$ Model 2 & 1.40 & $1.00-1.95$ & 0.051 \\
Model 3 & 1.60 & $1.11-2.29$ & 0.011 \\
$\quad$ Model 4 & 1.44 & $0.98-2.11$ & 0.065 \\
Infection-related mortality & & & \\
$\quad$ Unadjusted & 2.34 & $1.45-3.76$ & $<0.001$ \\
Model 1 & 1.68 & $1.04-2.72$ & 0.035 \\
$\quad$ Model 2 & 1.75 & $1.08-2.84$ & 0.024 \\
$\quad$ Model 3 & 2.00 & $1.18-3.40$ & 0.010 \\
Model 4 & 1.68 & $0.94-2.97$ & 0.078 \\
Stroke event after dialysis initiation & & & \\
$\quad$ Unadjusted & 2.61 & $1.70-4.01$ & $<0.001$ \\
Model 1 & 2.22 & $1.43-3.43$ & $<0.001$ \\
Model 2 & 2.14 & $1.38-3.31$ & $<0.001$ \\
Model 3 & 2.38 & $1.52-3.74$ & $<0.001$ \\
Model 4 & 2.23 & $1.41-3.54$ & $<0.001$ \\
\hline & & &
\end{tabular}

Model 1: Adjusted for age and gender.

Model 2: Model 1 plus DM, CAD, SBP, and DBP.

Model 3: Model 2 plus Hb, Alb, eGFR, and LDL-C.

Model 4: Model 3 plus BMI, CTR, loop diuretics, CCB, statin, and ACEIARB.

$\mathrm{HR}$, hazard ratio; CV, cardiovascular; DM, diabetes mellitus; CAD, coronary artery disease; SBP, systolic blood pressure; $\mathrm{DBP}$, diastolic blood pressure; Hb, hemoglobin; Alb, albumin; eGFR, estimated glomerular filtration rate; LDL-C, low density lipoprotein cholesterol; BMI, body mass index; CTR, cardiothoracic ratio; $\mathrm{CCB}$, calcium channel blocker; ACEI, angiotensin converting enzyme inhibitor; $A R B$, angiotensin receptor blocker.

Multivariate Analysis of Factors Affecting CV-related, Non-CV-related, and Infection-related Mortality and Stroke Event after Dialysis Initiation in theEntire Cohort

We evaluated the HRs of ischemic stroke for CV-related, non-CV related, and infection-related mortality and stroke event after dialysis initiation using a multivariate cox proportional analysis (Table 5). The history of ischemic stroke prior to dialysis initiation was associated with stroke event after dialysis initiation (HR 2.23, 95\% CI 1.41-3.54).
Comparison of Baseline Patient Characteristics and Laboratory Data between the 2 Groups in the PS-Matched Cohort

There were 182 patients in each group. Table 6 shows the baseline patient characteristics and laboratory data of the 2 groups after PS matching. There were no significant differences between the 2 groups, except for heart failure symptoms and the use of antiplatelet drugs.

\section{Comparison of Mortalities between the 2 Groups in the PS-matched Cohort}

There were 109 cases of all-cause mortality (ischemic stroke group: 71, non-stroke group: 38), CV-related mortality (ischemic stroke group: 25, non-stroke group: 20), non-CV-related mortality (ischemic stroke group: 46, non-stroke group: 18), infection-related mortality (ischemic stroke group: 21, non-stroke group: 8), and stroke event after dialysis initiation (ischemic stroke group: 24 events, non-stroke group: 10 events) during the follow-up period. Figure 2 shows the comparison of all-cause mortality between the 2 groups in the PSmatched cohort, and Figure 3 shows the comparison of $\mathrm{CV}$-related mortality, non-CV-related mortality, infection-related mortality, and stroke event after dialysis initiation. All-cause, non-CV-related, and infection-related mortality and stroke event after dialysis initiation were significantly higher in the ischemic stroke group after PS matching (log-rank test: $p<0.001,<0.001,0.002$, and 0.002 respectively).

\section{Comparison of All-cause mortality between the 2}

Groups after PS Matching with BI Score Stratification

Figure 4 shows the comparison of all-cause mortality between the stratified groups with a BI score of 100 and $<100$. Significant differences were observed in the cumulative survival between the 2 groups with a BI score of 100 $(p=0.003)$.

\section{Discussion/Conclusion}

The present study revealed that a history of stroke prior to dialysis initiation was associated with all-cause, nonCV-related, and infection-related mortality of incident dialysis patients. In addition, the mortality rate was higher in the ischemic stroke group among patients with ADL independence. The significant features of the present study are as follows: (1) we set the baseline at the time of dialysis initiation and (2) we compared the mortality be-
48

Nephron 2019;143:43-53 DOI: $10.1159 / 000500485$
Kojima et al. 
Table 6. Comparison of baseline characteristics laboratory data after PS matching

\begin{tabular}{|c|c|c|c|}
\hline Variables & $\begin{array}{l}\text { Ischemic stroke group } \\
(n=182)\end{array}$ & $\begin{array}{l}\text { Non-stroke group } \\
(n=182)\end{array}$ & $p$ value \\
\hline Age, years & $72.2(9.7)$ & $72.2(9.7)$ & 1.000 \\
\hline Gender, female, $n(\%)$ & $34(18.7)$ & $34(18.7)$ & 1.000 \\
\hline $\mathrm{DM}, n(\%)$ & $107(58.8)$ & $107(58.8)$ & 1.000 \\
\hline BMI, $\mathrm{kg} / \mathrm{m}^{2}$ & $22.7(3.6)$ & $22.9(3.2)$ & 0.541 \\
\hline $\mathrm{SBP}, \mathrm{mm} \mathrm{Hg}$ & $150(28)$ & $152(26)$ & 0.560 \\
\hline $\mathrm{DBP}, \mathrm{mm} \mathrm{Hg}$ & $74(15)$ & $75(15)$ & 0.369 \\
\hline History of CAD, $n(\%)$ & $25(13.7)$ & $25(13.7)$ & 1.000 \\
\hline Heart failure symptom, $n(\%)$ & $67(36.8)$ & $47(25.8)$ & 0.034 \\
\hline $\mathrm{CTR}, n(\%)$ & $56.3(7.1)$ & $55.4(7.4)$ & 0.248 \\
\hline $\mathrm{eGFR}, \mathrm{mL} / \mathrm{min} / 1.73 \mathrm{~m}^{2}$ & $5.91(2.5)$ & $5.63(1.9)$ & 0.224 \\
\hline Serum creatinine, $\mathrm{mg} / \mathrm{dL}$ & $8.37(2.82)$ & $8.63(2.75)$ & 0.374 \\
\hline BUN, mg/dL & $93.0(27.9)$ & $89.5(27.6)$ & 0.233 \\
\hline Hemoglobin, g/dL & $9.3(1.5)$ & $9.6(1.4)$ & 0.067 \\
\hline Serum albumin, g/dL & $3.16(0.58)$ & $3.17(0.51)$ & 0.970 \\
\hline Serum sodium, mEq/L & $138(5.0)$ & $138(3.8)$ & 0.398 \\
\hline Serum potassium, $\mathrm{mEq} / \mathrm{L}$ & $4.5(1.0)$ & $4.5(0.8)$ & 0.593 \\
\hline Serum uric acid, mg/dL & $8.7(2.2)$ & $8.8(2.3)$ & 0.791 \\
\hline Serum adjusted calcium, $\mathrm{mg} / \mathrm{dL}$ & $8.7(1.0)$ & $8.7(1.0)$ & 0.907 \\
\hline Serum phosphorus, mg/dL & $5.9(1.8)$ & $6.0(1.7)$ & 0.746 \\
\hline Serum LDL-C, mg/dL & $89(36)$ & $87(29)$ & 0.610 \\
\hline Serum HDL-C, mg/dL & $44(15)$ & $44(15)$ & 0.645 \\
\hline Serum triglyceride, $\mathrm{mg} / \mathrm{dL}$ & $117(61)$ & $121(67)$ & 0.613 \\
\hline Serum CRP, ng/mL* & $0.68(0.18-2.14)$ & $0.21(0.09-1.28)$ & 0.710 \\
\hline Intact PTH, pg/mL* & $258(147-387$ & $264(190-411)$ & 0.344 \\
\hline $\mathrm{HCO}_{3}{ }^{-} \mathrm{mmol} / \mathrm{L}$ & $20.1(5.2)$ & $19.8(4.3)$ & 0.605 \\
\hline Use of CCBs, $n(\%)$ & $144(79.1)$ & $150(82.4)$ & 0.506 \\
\hline Use of ACEIs/ARBs, $n(\%)$ & $107(58.8)$ & $103(56.6)$ & 0.750 \\
\hline Use of $\beta$-blockers, $n(\%)$ & $66(36.3)$ & $69(37.9)$ & 0.828 \\
\hline Use of anti-platelet agents, $n(\%)$ & $131(72.0)$ & $53(29.1)$ & $<0.001$ \\
\hline Use of Warfarin, $n(\%)$ & $19(10.4)$ & $14(7.7)$ & 0.466 \\
\hline Use of loop diuretics, $n(\%)$ & $120(65.9)$ & $125(68.7)$ & 0.655 \\
\hline Use of statins, $n(\%)$ & $79(43.4)$ & $61(33.5)$ & 0.067 \\
\hline Use of ESAs, $n(\%)$ & $17(90.6)$ & $17(90.7)$ & 1.000 \\
\hline
\end{tabular}

Values are expressed as mean (SD).

* Median (interquartile range).

PS, propensity score; BMI, body mass index; SBP, systolic blood pressure; DBP, diastolic blood pressure; CAD, coronary artery disease; CTR, cardiothoracic ratio; eGFR, estimated glomerular filtration rate; BUN, blood urea nitrogen; LDL-C, low-density lipoprotein cholesterol; HDL-C, high-density lipoprotein cholesterol; CRP, C-reactive protein; $\mathrm{PTH}$, parathyroid hormone; $\mathrm{CCB}$, calcium channel blocker; ACEI, angiotensin converting enzyme inhibitor; ARB, angiotensin receptor blocker; ESA, erythropoiesis stimulating agent; DM, diabetes mellitus.

tween the 2 groups after PS matching in order to adjust the background characteristics as necessary.

Previous reports indicated that the ischemic stroke risk factors reflected those of atherosclerosis $[15,16]$. In the present study, patients in the ischemic stroke group were significantly older with a greater number of men and higher DM comorbidity. However, there were no significant differences in serum lipid profiles between the 2 groups. In the general population, LDL-C correlates with atherosclerosis. However, previous reports have shown that lowering LDL-C level does not prevent atherosclerosis in dialysis patients [17]. Our observation that the ischemic stroke group did not show significant differences in serum lipid profiles is consistent with the findings of the previous report. Furthermore, we hypothesized that the decline in kidney function led to a poorer nutrition state in both groups; for this reason, hyperlipidemia, which potentially existed, might be concealed. 
Fig. 2. Comparison of all-cause mortality between the 2 groups in the PS-matched cohort. All-cause mortality was significantly higher in the ischemic stroke group (logrank test $p<0.001$ ).

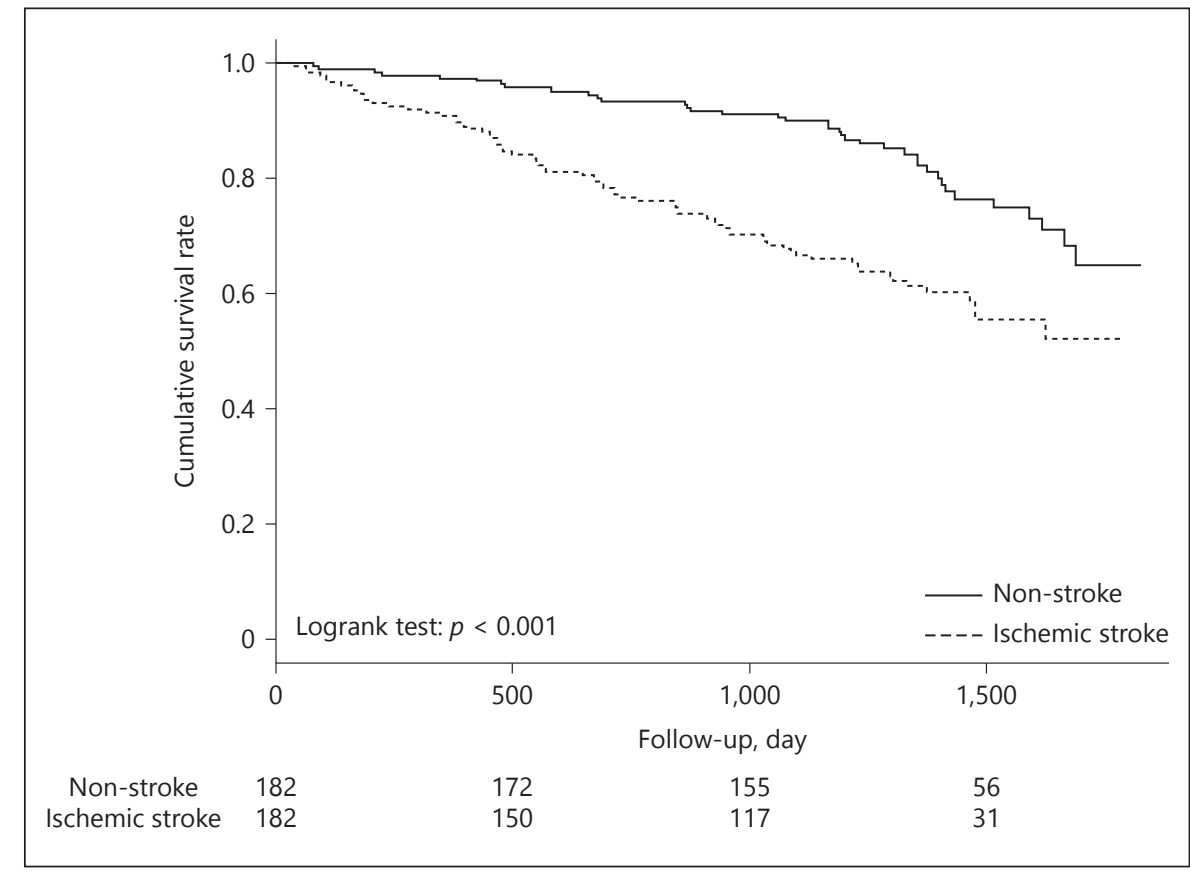

With regard to kidney function, eGFR was higher in the ischemic stroke group at the time of dialysis initiation. Early dialysis initiation is reportedly associated with better survival [18-24]. However, the Initiating Dialysis Early and Late study, which has a randomized controlled design, revealed that early dialysis initiation did not guarantee survival merit [25]. Yamagata et al. [26] showed that a higher eGFR at dialysis initiation was associated with a higher mortality rate during maintenance dialysis based on the Japanese Society for Dialysis Therapy registry data. In our view, earlier dialysis initiation with a high eGFR is more appropriate for the ischemic stroke group than for the non-stroke group, given the possible presence of critical conditions such as heart failure and over-hydration. Heart failure symptoms were significant in the entire cohort; these symptoms were in higher proportion in the ischemic stroke group. Besides, CTR in the ischemic stroke group was higher than that in the non-stroke group, but without significance, indicating that patients in the ischemic stroke group had more risk factors for $\mathrm{CV}$ disease at dialysis initiation, resulting in a higher incidence of mortality during maintenance dialysis.

We conducted PS matching for minimizing the differences in the baseline characteristics and laboratory data between the 2 groups. We found mortality, including allcause mortality, to be significantly higher in the ischemic stroke group even after PS matching. The present study demonstrated higher infection-related and non-CV-related mortalities in the ischemic stroke group. Generally, chronic inflammation and malnutrition were strongly associated with the onset and progress of CV disease. Sarcopenia, frailty, and the malnutrition-inflammation-atherosclerosis (MIA) syndrome are the main health problems in dialysis patients [27, 28]. Zimmermann et al. [29] showed that increased serum CRP levels are independent risk factors for CV disease. Stenvinkel et al. [30] showed that both inflammation and malnutrition are strongly associated with CV disease and arteriosclerosis and have described MIA syndrome. However, in our study, there were no significant differences in CRP and lipid profiles between the 2 groups, probably because the baseline was set at the time of dialysis initiation. Moreover, chronic inflammation and malnutrition caused by uremia, which were maximized at dialysis initiation, may have concealed the preexisting chronic inflammation and malnutrition. However, the relationship between lipid profiles and cerebral infarction remains to be clarified. Atherosclerotic cerebral infarction that accounts for about a quarter of cerebral infarction cases in Japan was reported to be positively associated with LDL-C and total cholesterol levels $[31,32]$.

All-cause mortality was higher in the ischemic stroke group stratified according to BI scores. We compared ADL independence between the 2 groups, with BI score of 100 versus score of $<100$. This allowed the 2 groups to 


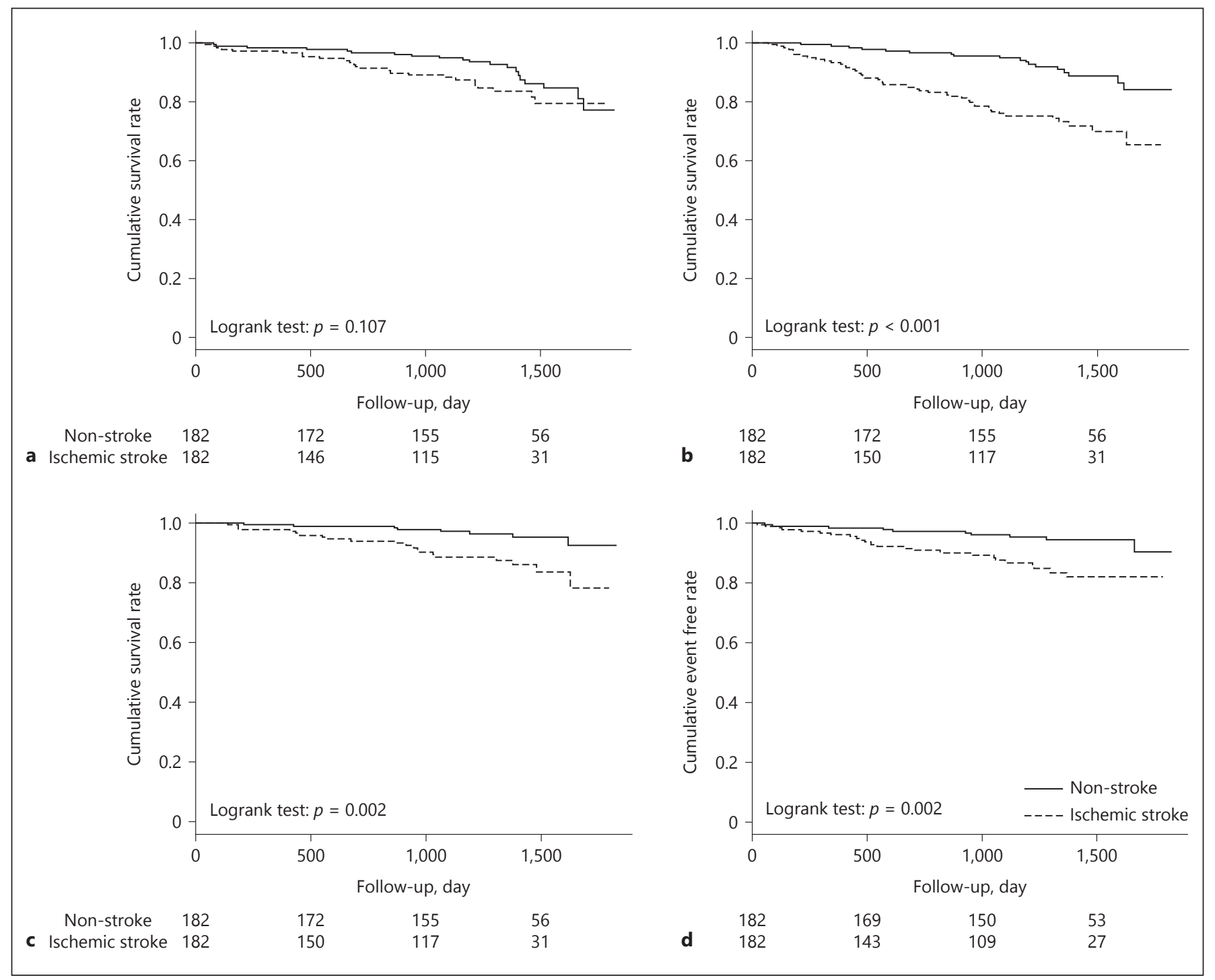

Fig. 3. Comparison of mortality between the 2 groups in the PSmatched cohort. a CV-related mortality, (b) non-CV-related mortality, (c) infection-related mortality, and (d) stroke event after dialysis initiation, Non-CV-related mortality, infection-related

be compared based on whether or not ADL was independent. Based on the previously mentioned results, the history of stroke in incident dialysis patients was significantly associated with higher mortality in the ADL-dependent group. We supposed that the poor general health condition caused by the underlying sarcopenia, frailty, and MIA syndrome resulted in a high mortality rate among patients in the ischemic stroke group; nevertheless, uremia was improved with dialysis treatment [33].

We compared only the ischemic stroke and non-stroke patients and excluded those with hemorrhagic stroke be-

Stroke and Mortality in Incident Dialysis Patients mortality, stroke event after dialysis initiation were significantly higher in the stroke group (log-rank test: $p<0.001, p=0.002$, and $p=0.002$ respectively).

cause of the difference between the cause of ischemic stroke and cerebral hemorrhage as well as because there were fewer patients with a history of cerebral hemorrhage than with a history of ischemic stroke in the cohort. Most cerebral hemorrhage cases were due to high blood pressure and had a poor prognosis.

The present study has several limitations. First, it is an observational analysis, and there were some differences in the baseline characteristics and laboratory data between the 2 groups. Therefore, residual confounding bias may have occurred, and although we used PS-matched cohorts of the 


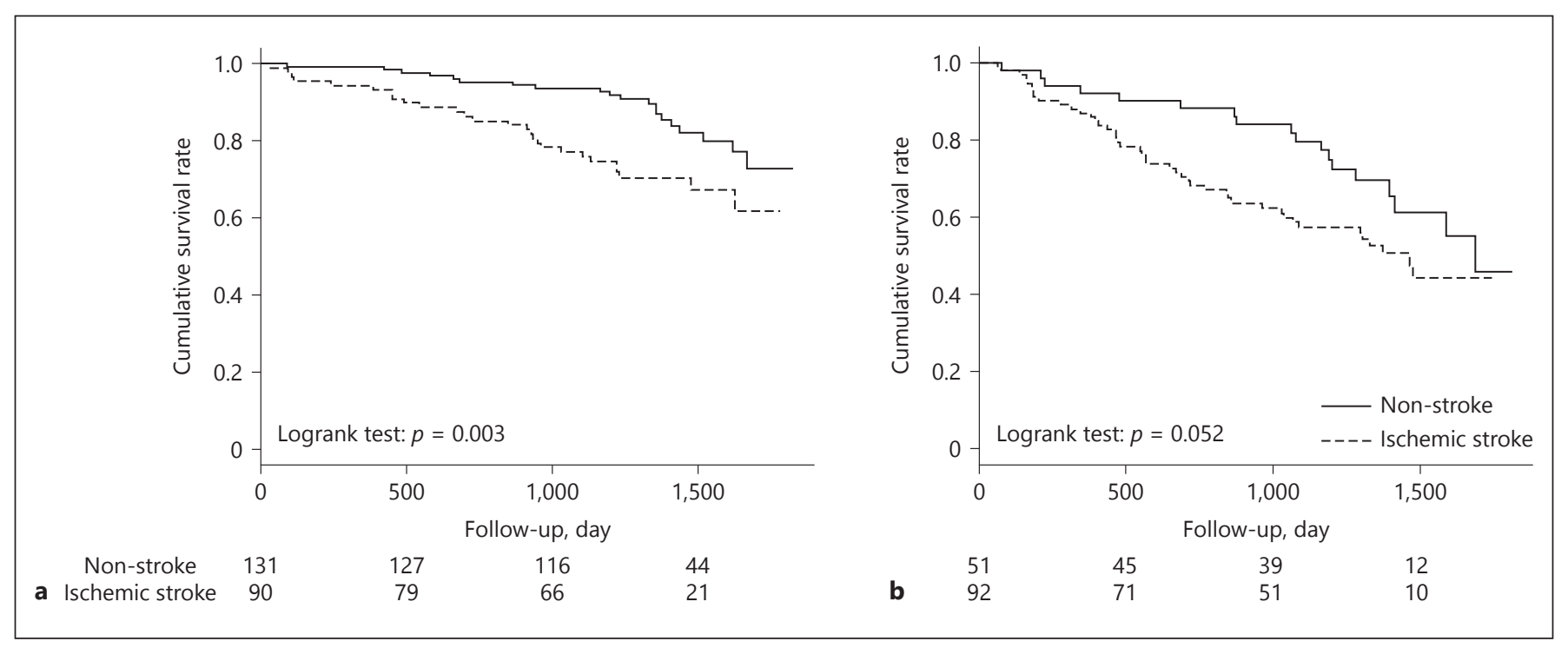

Fig. 4. Comparison of all-cause mortality between the 2 groups after PS matching with BI score stratification. All-cause mortality was significantly higher in the ischemic stroke group regardless of physical function as indicated by the BI score $(p=0.003$ and $p=$ 0.052 , respectively). (a) The group with a BI score of 100 , (b) the group with a BI score $<100$.
2 groups to minimize this problem, we were unable to match the 2 groups entirely. Second, we could not determine the ischemic stroke origins, such as lacunar stroke, aortic atheroma, or embolization. Finally, unknown confounding factors may not have been included in the analysis.

The significant features of the present study are as follows: (1) we set the baseline at the time of dialysis initiation and (2) we compared the mortality between the 2 groups after PS matching in order to adjust the background characteristics as necessary.

Based on the findings of the present study, a history of ischemic stroke prior to dialysis initiation is associated with all-cause, non-CV-related, and infection-related mortality rates and stroke event after dialysis initiation during maintenance dialysis.

\section{Acknowledgment}

We acknowledge the support provided by the investigators and members of the AICOPP. The Aichi Kidney Foundation funded this study.

\section{Statement of Ethics}

This study was conducted by following Ethical guidelines for Clinical Research by the Japanese Ministry of Health, Labor, and Welfare (created July 30, 2003; full revision December 28, 2004; full revision July 31, 2008) and the Helsinki Declaration (revised 2013) and was approved by the clinical research Ethics Committees at each AICOPP group facility (the approval number: 20110823-3). The subjects received oral and written explanations of the purpose of the study and provided their consent in writing. Trial registration is UMIN 7096 And it was registered on January $18,2012$.

\section{Disclosure Statement}

Y.Y. has received research support grants from Otsuka Pharmaceutical Co., Ltd., Kyowa Hakko Kirin Co., Ltd., and Chugai Pharmaceutical Co., Ltd. D.I. has received lecture fee from Ono Pharmaceutical Co., Ltd., Kyowa Hakko Kirin Co., Ltd. Other authors have nothing to declare.

\section{Funding Sources}

The Aichi Kidney Foundation partially funded this study.

\section{Author Contributions}

D.I. and S.K. participated in the design of the study and interpretation of data. M.K., S.K. and D.I. participated in writing the manuscript. All authors were involved in drafting, reviewing, and approving the final manuscript.
Kojima et al. 


\section{References}

1 Sarnak MJ, Levey AS, Schoolwerth AC, Coresh J, Culleton B, Hamm LL, et al.; American Heart Association Councils on Kidney in Cardiovascular Disease, High Blood Pressure Research, Clinical Cardiology, and Epidemiology and Prevention. Kidney disease as a risk factor for development of cardiovascular disease: a statement from the American Heart Association Councils on Kidney in Cardiovascular Disease, High Blood Pressure Research, Clinical Cardiology, and Epidemiology and Prevention. Circulation. 2003 Oct; 108(17):2154-69.

2 Nakayama M, Metoki H, Terawaki H, Ohkubo T, Kikuya M, Sato T, et al. Kidney dysfunction as a risk factor for first symptomatic stroke events in a general Japanese population-the Ohasama study. Nephrol Dial Transplant. 2007 Jul;22(7):1910-5.

3 Anavekar NS, McMurray JJ, Velazquez EJ, Solomon SD, Kober L, Rouleau JL, et al. Relation between renal dysfunction and cardiovascular outcomes after myocardial infarction. N Engl J Med. 2004 Sep;351(13):128595.

4 Go AS, Chertow GM, Fan D, McCulloch CE, Hsu CY. Chronic Kidney Disease and the Risks of Death, Cardiovascular Events, and Hospitalization. N Engl J Med. 2004 Sep; 351(13):1296-305.

5 Kuwahara M, Takehara E, Sasaki Y, Azetsu H, Kusaka K, Shikuma S, et al. Effects of Cardiovascular Events on End-Stage Renal Disease and Mortality in Patients With Chronic Kidney Disease Before Dialysis. Ther Apher Dial. 2016 Feb;20(1):12-9.

6 Arnold J, Sims D, Ferro CJ. Modulation of stroke risk in chronic kidney disease. Clin Kidney J. 2016 Feb;9(1):29-38.

7 Ovbiagele B, Schwamm LH, Smith EE, GrauSepulveda MV, Saver JL, Bhatt DL, et al. Patterns of care quality and prognosis among hospitalized ischemic stroke patients with chronic kidney disease. J Am Heart Assoc. 2014 Jun;3(3):e000905.

8 Del Fabbro P, Luthi JC, Carrera E, Michel P, Burnier M, Burnand B. Anemia and chronic kidney disease are potential risk factors for mortality in stroke patients: a historic cohort study. BMC Nephrol. 2010 Oct;11(1):27.

9 Saeed F, Kousar N, Qureshi K, Laurence TN. A review of risk factors for stroke in patients with chronic kidney disease. J Vasc Interv Neurol. 2009 Jan;2(1):126-31.

10 Tong J, Liu M, Li H, Luo Z, Zhong X, Huang J, et al. Mortality and Associated Risk Factors in Dialysis Patients with Cardiovascular Disease. Kidney Blood Press Res. 2016;41(4):479-87.
11 Inaguma D, Koide S, Takahashi K, Hayashi $\mathrm{H}$, Hasegawa M, Yuzawa Y. Relationship between history of coronary heart disease at dialysis initiation and onset of events associated with heart disease: a propensity-matched analysis of a prospective cohort study. BMC Nephrol. 2017 Feb;18(1):79.

12 Hishida M, Tamai H, Morinaga T, Maekawa M, Aoki T, Tomida H, et al. Aichi cohort study of the prognosis in patients newly initiated into dialysis (AICOPP): baseline characteristics and trends observed in diabetic nephropathy. Clin Exp Nephrol. 2016 Oct;20(5):795-807.

13 Quinn TJ, Langhorne P, Stott DJ. Barthel index for stroke trials: development, properties, and application. Stroke. 2011 Apr;42(4): 1146-51.

14 Kanda Y. Investigation of the freely available easy-to-use software 'EZR' for medical statistics. Bone Marrow Transplant. 2013 Mar; 48(3):452-8.

15 Boysen G, Nyboe J, Appleyard M, Sørensen PS, Boas J, Somnier F, et al. Stroke incidence and risk factors for stroke in Copenhagen, Denmark. Stroke. 1988 Nov;19(11):134553.

16 Iso H, Jacobs DR Jr, Wentworth D, Neaton JD, Cohen JD. Serum cholesterol levels and six-year mortality from stroke in 350,977 men screened for the multiple risk factor intervention trial. N Engl J Med. 1989 Apr;320(14): 904-10.

17 Ferro CJ, Mark PB, Kanbay M, Sarafidis P, Heine GH, Rossignol P, et al. Author Correction: lipid management in patients with chronic kidney disease. Nat Rev Nephrol. 2019 Feb;15(2):121.

18 Bonomini V, Feletti C, Scolari MP, Stefoni S. Benefits of early initiation of dialysis. Kidney Int Suppl. 1985 Dec;17:S57-9.

19 Perrone RD, Madias NE, Levey AS. Serum creatinine as an index of renal function: new insights into old concepts. Clin Chem. 1992 Oct;38(10):1933-53.

20 Dombros N, Dratwa M, Feriani M, Gokal R, Heimbürger O, Krediet R, et al.; EBPG Expert Group on Peritoneal Dialysis. European best practice guidelines for peritoneal dialysis. 2 The initiation of dialysis. Nephrol Dial Transplant. 2005 Dec;20(Suppl 9):ix3-7.

21 Churchill DN. An evidence-based approach to earlier initiation of dialysis. Am J Kidney Dis. 1997 Dec;30(6):899-906.

22 Tattersall J, Greenwood R, Farrington K. Urea kinetics and when to commence dialysis. Am J Nephrol. 1995;15(4):283-9.

23 Hakim RM, Lazarus JM. Initiation of dialysis. J Am Soc Nephrol. 1995 Nov;6(5):1319-28.
24 Kim SG, Kim NH. The effect of residual renal function at the initiation of dialysis on patient survival. Korean J Intern Med (Korean Assoc Intern Med). 2009 Mar;24(1):55-62.

25 Cooper BA, Branley P, Bulfone L, Collins JF, Craig JC, Fraenkel MB, et al.; IDEAL Study. A randomized, controlled trial of early versus late initiation of dialysis. N Engl J Med. 2010 Aug;363(7):609-19.

26 Yamagata K, Nakai S, Masakane I, Hanafusa N, Iseki K, Tsubakihara Y; Committee of Renal Data Registry of the Japanese Society for Dialysis Therapy. Ideal timing and predialysis nephrology care duration for dialysis initiation: from analysis of Japanese dialysis initiation survey. Ther Apher Dial. 2012 Feb;16(1): 54-62.

27 Johansen KL, Chertow GM, Jin C, Kutner NG. Significance of frailty among dialysis patients. J Am Soc Nephrol. 2007 Nov; 18(11): 2960-7.

28 Sueta D, Hokimoto S, Sakamoto K, Akasaka T, Tabata N, Kaikita K, et al.; Multi-center Study of Hemodialysis Patients Undergoing Invasive Cardiovascular Procedures Study Investigators. Validation of the high mortality rate of Malnutrition-Inflammation-Atherosclerosis syndrome: Community-based observational study. Int J Cardiol. 2017 Mar;230: 97-102.

29 Zimmermann J, Herrlinger S, Pruy A, Metzger T, Wanner C. Inflammation enhances cardiovascular risk and mortality in hemodialysis patients. Kidney Int. 1999 Feb;55(2): 648-58.

30 Stenvinkel P, Heimbürger O, Lindholm B, Kaysen GA, Bergström J. Are there two types of malnutrition in chronic renal failure? Evidence for relationships between malnutrition, inflammation and atherosclerosis (MIA syndrome). Nephrol Dial Transplant. 2000 Jul; 15(7):953-60.

31 Imamura $\mathrm{T}$, Doi $\mathrm{Y}$, Arima $\mathrm{H}$, Yonemoto $\mathrm{K}$, Hata J, Kubo M, et al. LDL cholesterol and the development of stroke subtypes and coronary heart disease in a general Japanese population: the Hisayama study. Stroke. 2009 Feb; 40(2):382-8.

32 Cui R, Iso H, Yamagishi K, Saito I, Kokubo Y, Inoue M, et al.; JPHC Study Group. High serum total cholesterol levels is a risk factor of ischemic stroke for general Japanese population: the JPHC study. Atherosclerosis. 2012 Apr;221(2):565-9.

33 Musso CG, Jauregui JR, Macías Núñez JF. Frailty phenotype and chronic kidney disease: a review of the literature. Int Urol Nephrol. 2015 Nov;47(11):1801-7. 